\title{
DISTRIBUIÇÃO ESPACIAL DE IDOSOS DE ACORDO COM MENORES ESCORES DE QUALIDADE DE VIDA ${ }^{1}$
}

\author{
Darlene Mara dos Santos Tavares², Flavia Aparecida Dias ${ }^{3}$
}

\footnotetext{
${ }^{1}$ Artigo decorrente de projeto financiado pela Fundação de Amparo a Pesquisa do Estado de Minas Gerais. Processo N APQ05909-07.

${ }^{2}$ Doutora em Enfermagem. Professora Associado do Departamento de Enfermagem em Educação e Saúde Comunitária do Curso de Graduação em Enfermagem da Universidade Federal do Triângulo Mineiro (UFTM). Minas Gerais, Brasil. E-mail: darlenetavares@enfermagem.uftm.edu.br

${ }^{3}$ Mestranda do Programa de Pós-Graduação Stricto sensu em Atenção à Saúde da UFTM. Minas Gerais, Brasil. E-mail: flaviadias_ura@yahoo.com.br
}

RESUMO: Esta pesquisa objetivou descrever o perfil sociodemográfico e a qualidade de vida dos idosos, e mapear e identificar áreas de menores escores de qualidade de vida. Estudo descritivo, transversal e observacional. Foram entrevistados 2.142 idosos no domicílio, com instrumentos WHOQOL-BREF e WHOQOL-OLD, no período de agosto a dezembro de 2008. Para análise dos dados utilizou-se frequência simples e Kernel estimation. A maioria era do sexo feminino, até 70 anos, casados ou morava com companheiro, e renda de um salário mínimo. A qualidade de vida evidenciou maiores escores para o domínio relações sociais e a faceta funcionamento dos sentidos, enquanto os menores estavam relacionados ao físico e à autonomia. A distribuição espacial dos menores escores de qualidade de vida apresentou aglomerados nas regiões periféricas do município. O conhecimento do perfil socioeconômico populacional e a distribuição espacial possibilitam o desenvolvimento de estratégias baseadas nas reais necessidades dos idosos.

DESCRITORES: Envelhecimento. Idoso. Enfermagem geriátrica. Qualidade de vida. Sistemas de informação geográfica.

\section{ELDERY SPATIAL DISTRIBUTION ACCORDING TO LOWER QUALITY OF LIFE SCORES}

\begin{abstract}
This study aimed to describe the socio-demographic profile and quality of life for seniors in Brazil; as well as to map and identify areas of lower quality of life scores. This is a cross-sectional, descriptive, and observational study which interviewed 2142 elderly in their homes, using the WHOQOL-BREF and WHOQOL-OLD instruments between August and December of 2008. Data was analyzed using simple frequency and kernel estimation. The majority of the sample was female, less than 70 years old, married or living with a partner, and earned minimum wage incomes. The quality of life showed higher scores in the social relationships domain and the functional facet of their senses, with the lowest scores related to physical aging and autonomy. Spatial distribution of the lower quality of life scores presented clusters in the outskirts of respective cities. Better knowledge of socioeconomic profiles of populations and spatial distribution allow for strategies based on the real needs of the elderly to be developed.
\end{abstract}

DESCRIPTOR: Aging. Aged. Geriatric nursing. Quality of life. Geographic information systems.

\section{DISTRIBUCIÓN ESPACIAL DE LOS ANCIANOS CON RELACIÓN A LA MENOR PUNTUACIÓN DE CALIDAD DE VIDA}

\begin{abstract}
RESUMEN: Esta investigación tuvo como objetivo describir el perfil sociodemográfico y la calidad de vida de los ancianos; e identificar y cartografiar las áreas que presentan menores resultados de puntuación con relación a la calidad de vida. Es un estudio descriptivo, transversal y de observación. Se entrevistaron 2.142 ancianos en sus hogares, con los instrumentos WHOQOL-BREF y WHOQOLOLD en el período de agosto a diciembre de 2008. Para el análisis de los datos se utilizó la frecuencia simple y la estimación vía Kernel. La mayoría eran mujeres, de hasta 70 años, casadas o vivían con una pareja, y con ingresos de un salario mínimo. La calidad de vida mostró puntuaciones más altas para el dominio relaciones sociales y las facetas de funcionamiento de los sentidos, mientras que la puntuación menor se relacionaba con lo físico y la autonomía. La distribución espacial de los puntajes más bajos para la calidad de vida se presentan en las áreas periféricas de la ciudad. El conocimiento del perfil socioeconómico de la población y la distribución espacial permitirá el desarrollo de estrategias basadas en las necesidades reales de los ancianos.
\end{abstract}

DESCRIPTORES: Envejecimiento. Anciano. Enfermería geriátrica. Calidad de vida. Sistemas de información geográfica. 


\section{INTRODUÇÃO}

O envelhecimento populacional no Brasil ocorre de forma acelerada. O número de idosos passou de 3 milhões, em 1960, para 7 milhões, em 1975, e 20 milhões em 2008. Um aumento de aproximadamente $700 \%$ em menos de 50 anos. ${ }^{1}$

Estima-se que a população idosa no país seja de $11,1 \% .{ }^{2}$ No município de Uberaba, local em que foi realizado este estudo, a população idosa corresponde a $12,1 \%{ }^{3}$ Estes dados evidenciam a necessidade de desenvolver pesquisas direcionadas à esta população, visando compreender o processo de envelhecimento populacional, considerando suas características, bem como a sua distribuição regional.

O envelhecimento humano só pode ser considerado uma real conquista, para a sociedade, na medida em que se agregue qualidade aos anos vividos. Deste modo, a atenção destinada aos idosos deve considerar a capacidade funcional, a autonomia, a participação social, as necessidades de cuidado e satisfação pessoal, assim como, a manutenção da inserção social e de boas condições de saúde. ${ }^{1-4}$ Todos estes aspectos podem impactar de forma positiva ou negativa na qualidade de vida do idoso, guardando relação com o contexto social e familiar, a sua história de vida e a organização da atenção à saúde.

Neste estudo será adotado o conceito de qualidade de vida proposto por um grupo de estudiosos da Organização Mundial de Saúde (OMS). É considerado subjetivo, multidimensional, com aspectos positivos e negativos: "percepção do indivíduo de sua posição na vida no contexto da cultura e sistema de valores nos quais ele vive e em relação aos seus objetivos, expectativas, padrões e preocupações" .5:1405

À identificação do escore de qualidade de vida do idoso soma-se a necessidade de conhecer o seu padrão de distribuição espacial, dada a possibilidade de associação com as diferenças sociais e territoriais. O espaço é produto e produtor de diferenciações sociais e ambientais, tendo reflexos sobre a saúde e a qualidade de vida da população. Os escores de qualidade de vida tendem a se agrupar espacialmente de acordo com as similaridades culturais e socioeconômicas. ${ }^{6}$ Nesta perspectiva, é mister repensar ferramentas que contribuam para melhor alocação de recursos e ações direcionadas às especificidades regionais da população.
A aplicação dos Sistemas de Informação Geográfica (SIG) tem sido descrita como ferramenta útil para reorientação de programas gerontológicos e políticas de saúde. ${ }^{7}$ Porém, a literatura científica sobre o tema ainda é escassa. Pesquisa conduzida com idosos observou que o padrão de distribuição espacial era formado por agrupamentos nos setores censitários segundo o modo como definem qualidade de vida. ${ }^{8}$

A tendência sobre estudos de qualidade de vida, saúde e análise espacial tem sido apontada por meio do uso de indicadores, como Índice de Desenvolvimento Humano (IDH), que visa desvelar a ocorrência de desigualdades no espaço. A localização espacial permite evidenciar as diferenças de acesso aos recursos e oportunidades de vida. ${ }^{6}$

Reconhecendo que a qualidade de vida possui distintas dimensões, pretende-se obter tais informações utilizando-se de instrumento específico destinado aos idosos e visualizar áreas de menores escores expressos em mapas temáticos. Assim, tem-se a possibilidade de fornecer subsídios para o planejamento de ações em saúde na atenção à população idosa, de acordo com as especificidades de cada localidade.

Posto isso, os objetivos deste estudo foram: descrever o perfil sociodemográfico e a qualidade de vida dos idosos, e mapear e identificar possíveis áreas de concentração de menores escores de qualidade de vida.

\section{MÉTODO}

Trata-se de um estudo descritivo, transversal e observacional, desenvolvido na zona urbana do município de Uberaba-MG.

Para a definição da população foi utilizada a amostra populacional realizada em estudo anterior pelo Núcleo de Pesquisa em Saúde Coletiva. ${ }^{9}$ O cálculo do tamanho amostral utilizou $95 \%$ de confiança, $80 \%$ de poder do teste, margem de erro de $4 \%$ para as estimativas intervalares e uma proporção estimada de $\mathrm{p}=0,5$ para as proporções de interesse.

Nesta pesquisa, a amostra foi constituída pelos idosos que atenderam aos critérios de inclusão: ter 60 anos ou mais de idade; ter obtido pontuação mínima de 13 pontos na avaliação cognitiva ${ }^{* *}$; ambos os sexos, morar na zona urbana no

* A avaliação cognitiva, composta por seis questões, visa identificar a preservação da memória recente. O somatório das questões varia de zero a 19 pontos, tendo o número treze como ponto de corte. Desta forma, o idoso tem condições de responder as questões propostas pelo estudo. 
município de Uberaba-MG e concordar participar da pesquisa. Desta forma, a amostra populacional foi constituída por 2.142 idosos.

Os dados foram coletados no domicílio, utilizando-se de instrumento semiestruturado para a obtenção das variáveis sociodemográficas e a qualidade de vida foi mensurada pelo WHOQOL-BREF (instrumento genérico) ${ }^{10} \mathrm{e} W H O Q O L-O L D$ (específico). ${ }^{11}$ As atividades de campo, realizadas por uma equipe de 12 entrevistadores selecionados e capacitados para o seu desenvolvimento, ocorreram de agosto a dezembro de 2008.

Optou-se pela entrevista direta na aplicação dos instrumentos de qualidade de vida, WHOQOL-BREF ${ }^{10}$ e WHOQOL-OLD ${ }^{11}$, que podem ser autoaplicáveis, em razão da possível dificuldade de leitura, problemas visuais e analfabetismo entre os idosos.

O módulo WHOQOL-BREF é composto por 26 questões, no qual as duas primeiras são genéricas e compõem a qualidade de vida geral. Esta versão abreviada é composta por quatro domínios. ${ }^{10}$ Já o módulo WHOQOL-OLD tem 24 itens atribuídos em um escore total e seis facetas. ${ }^{11}$

As variáveis estudadas foram: sociodemográficas (sexo, escolaridade, faixa etária e renda) e qualidade de vida (domínios do WHOQOL-BREF: físico, psicológico, relações sociais e meio ambiente; e facetas do WHOQOL-OLD: funcionamento dos sentidos; autonomia, atividades passadas, presentes e futuras, participação social, morte e morrer e intimidade).

Foi construída planilha de dados eletrônica, no programa $\mathrm{Excel}^{\circledR}$, versão 2003, e os dados coletados foram processados em microcomputador, por duas pessoas, em dupla entrada. Procedeu-se à consistência dos campos e os dados inconsistentes foram verificados na entrevista original e corrigidos.

Para a análise dos dados de qualidade de vida foi utilizado o software Statiscal Package for Social Sciences (SPSS), versão 17.0, e para a análise espacial, os programas MapInfo Professional versão 9.5 e Terraview versão 3.3.1.

Cada domínio do WHOQOL-BREF e faceta do WHOQOL-OLD foi analisado isoladamente com suas respectivas sintaxes. Os maiores escores correspondem a maior qualidade de vida. Os dados sociodemográficos foram analisados por meio de distribuição de frequência simples.

Para mapeamento da qualidade de vida, foram utilizadas as coordenadas Universal Transversa
Mercator (UTM), modelo da Terra (SAD-69), coletadas pelo programa Google Earth. Os endereços que não foram encontrados neste programa foram coletados através de equipamento apropriado do tipo Sistema de Posicionamento Global (GPS), das marcas Juno ST, PROXR e PROXRS.

As coordenadas planas (Hemisfério Sul) foram: X1: $184.780,48 \mathrm{~m}, \mathrm{X} 2: 196.450,74 \mathrm{~m}, \mathrm{Y1}$ : 7.808.333,18 m, e Y2: 7.814.259,92 m.

Os menores escores de qualidade de vida em cada domínio e faceta foram identificados através do percentil 10, que forneceu os $10 \%$ dos menores valores.

Para estimar a intensidade dos eventos utilizou-se o Kernel estimation com raio adaptativo da função quártica. Trata-se de uma técnica de interpolação exploratória que gera uma superfície para a identificação visual de áreas com maior densidade de eventos indicando aglomeração em uma distribuição espacial ${ }^{12}$. As áreas com taxas mais elevadas foram representadas por tons mais escuros. Cada célula equivale a $250 \mathrm{~m}$.

Este projeto foi aprovado pelo Comitê de Ética em Pesquisa com Seres Humanos da Universidade Federal do Triângulo Mineiro, protocolo ${ }^{0}$ 897. Os sujeitos desta pesquisa foram contactados em seus domicílios, aos quais foram apresentados os objetivos, o Termo de Consentimento Livre e Esclarecido e oferecidas as informações pertinentes. Somente após a anuência do entrevistado e assinatura do referido Termo foi conduzida a entrevista.

\section{RESULTADOS E DISCUSSÃO}

Na tabela 1, apresentam-se as variáveis sociodemográficas da população estudada.

Tabela 1 - Distribuição de frequência das variáveis sociodemográficas dos idosos. Uberaba-MG, 2010

(Continua)

\begin{tabular}{lrc}
\hline Variáveis & $\mathbf{n}$ & $\mathbf{\%}$ \\
\hline Sexo & & \\
Masculino & 804 & 37,5 \\
Feminino & 1338 & 62,4 \\
Faixa etária & & \\
60 -70 & 994 & 46,4 \\
70 -80 & 822 & 38,4 \\
80 ou mais & 326 & 15,2 \\
Estado conjugal & & \\
\hline
\end{tabular}




\begin{tabular}{|c|c|c|}
\hline \multirow[b]{2}{*}{ Variáveis } & \multicolumn{2}{|c|}{ (Conclusão) } \\
\hline & $\mathbf{n}$ & $\%$ \\
\hline Nunca se casou/morou com companheiro & 110 & 5,1 \\
\hline Viúvo & 796 & 37,1 \\
\hline Separado/desquitado/divorciado & 188 & 8,8 \\
\hline Casados ou moram com companheiro & 1046 & 48,9 \\
\hline \multicolumn{3}{|l|}{ Escolaridade (em anos) } \\
\hline Nenhum & 426 & 19,9 \\
\hline $1 \mid-4$ & 684 & 31,9 \\
\hline 4 -8 & 705 & 32,9 \\
\hline 8 anos & 95 & 4,4 \\
\hline 9 e mais & 215 & 10,0 \\
\hline \multicolumn{3}{|l|}{ Renda individual (salário mínimo) ${ }^{\dagger}$} \\
\hline Nenhuma & 224 & 10,5 \\
\hline$<1$ & 24 & 1,1 \\
\hline 1 & 1179 & 55,1 \\
\hline 1$\} 3$ & 577 & 26,9 \\
\hline $3-5$ & 84 & 3,9 \\
\hline 5 e mais & 40 & 1,9 \\
\hline
\end{tabular}

A maioria era do sexo feminino $(62,4 \%)$. Percentuais semelhantes foram encontrados em pesquisas conduzidas no mesmo município do presente estudo $(64 \%)^{15}$ e entre idosos selecionados em domicílios em Botucatu-SP (59,7\%). ${ }^{16}$

O sexo feminino possui maior longevidade relacionada à maior mortalidade masculina brasileira nas últimas décadas, que tem se apresentado alta com valores acima dos padrões internacionais. No Brasil, em 2000, para cada 100 mulheres idosas, havia 81 homens idosos e, em 2025, espera-se que esse número caia para $74 .{ }^{17}$

Estes dados evidenciam que os serviços de saúde devem considerar a maior proporção de mulheres idosas nas suas ações. Contudo, devem desenvolver, também, estratégias visando o cuidado à saúde do homem, com o objetivo de diminuir a morbimortalidade.

Observou-se predomínio da faixa etária de $60-70$ anos (46,4\%). Estes dados corroboram com a investigação realizada com idosos, em que $56,6 \%$ tinham entre 60 a 69 anos. ${ }^{18}$

Quanto ao estado conjugal, 48,9\% eram casados ou moravam com companheiro. Percentuais semelhantes ao encontrado em outros estudos. ${ }^{15-16,18-19}$ Porém, investigação realizada entre idosos cadastrados no Programa Saúde da Família
(PSF) destacou percentual superior ao encontrado nesta pesquisa $(62,6 \%) .{ }^{20}$ Destaca-se que $37,1 \%$ eram viúvos, denotando a necessidade do profissional de saúde identificar o apoio familiar a esse idoso.

Referente à escolaridade, prevaleceu de quatro a oito anos de estudo (32,8\%), seguido por de um a quatro anos $(31,9 \%)$. Investigação realizada entre idosos evidenciou que 71,1\% têm menos de quatro anos de estudo ${ }^{20}$, percentual superior ao encontrado neste estudo. A pouca escolaridade dos idosos reflete a desigualdade social e as políticas de educação das décadas de 1930 e 1940, quando o acesso à escola era muito restrito. ${ }^{21} \mathrm{~A}$ baixa escolaridade pode influenciar no autocuidado à saúde, desta forma, o enfermeiro deve considerar esta situação, verificando a compreensão do idoso acerca das orientações recebidas.

Observou-se que a maioria recebe um salário mínimo (55,1\%); resultado que é condizente com inquérito realizado no mesmo município $(51,9 \%)^{15}$ e em Fortaleza-CE $(62,8 \%) \cdot{ }^{18}$ A renda obtida está abaixo da encontrada entre idosos moradores da periferia, que recebiam de um a dois salários mínimos $(66,2 \%){ }^{22}$

Ao se considerar o perfil sociodemográfico dos idosos na implementação do cuidado em enfermagem é possível desenvolver ações individualizadas, no contexto coletivo, e de acordo com as especificidades apresentadas. Desta forma, o cuidado será mais efetivo e humanizado.

Em relação à autoavaliação da qualidade de vida, a maioria dos idosos respondeu como boa $(67,2 \%)$, seguido por nem boa, nem ruim $(22 \%)$; resultado semelhante ao obtido em outro estudo, em que a maioria dos idosos classificou a qualidade de vida como boa ( $46 \%$ ) e nem boa, nem ruim ( $32 \%) .{ }^{23}$

$\mathrm{Na}$ autosatisfação com a saúde, $64,4 \%$ consideraram-se satisfeitos e (16,4\%) nem satisfeitos, nem insatisfeitos. Pesquisa evidenciou que a maioria dos idosos estava satisfeita com sua vida em geral e em aspectos específicos. O grau de satisfação associou-se com: conforto domiciliar, valorização do lazer como qualidade de vida, acordar bem pela manhã, não referir solidão e fazer três ou mais refeições. ${ }^{16}$

O maior escore de qualidade de vida foi para o domínio relações sociais $(68,8)$; resultado semelhante ao encontrado em inquérito conduzido com idosos. ${ }^{20}$ Este resultado denota que as relações pessoais e apoio social impactam positivamente na qualidade de vida dos idosos. ${ }^{10}$

O domínio físico apresentou o menor escore $(59,5)$ de qualidade de vida, divergindo de inves- 
tigação com idosos na comunidade, na qual foi domínio psicológico $(55,9) \cdot{ }^{24} \mathrm{O}$ enfermeiro pode identificar nas consultas de enfermagem, bem como, nas visitas domiciliares, quais os fatores que estão influenciando negativamente no aspecto físico do idoso. Tais fatores podem estar relacionados à diminuição de energia, dificuldades na realização das atividades cotidianas e dependência de medicação e tratamentos, uma vez que este aspectos são avaliados neste domínio. ${ }^{10}$

A qualidade de vida mensurada pelo $\mathrm{WHO}-$ QOL-OLD evidenciou maior escore de qualidade de vida na faceta funcionamento dos sentidos $(79,9)$, divergindo dos resultados observados entre idosos de Erechim-RS' ${ }^{25}(72,1)$ e de Porto Alegre ${ }^{26}$ $(76,2)$, em que foi para a participação social.

A faceta autonomia $(60,4)$ obteve o menor escore de qualidade de vida, corroborando com os estudos conduzidos com idosos em Erechim-RS ${ }^{25}$ $(66,4)$ e Porto Alegre ${ }^{26}(36,5)$. Nesta perspectiva, observa-se a necessidade do enfermeiro discutir com os familiares o processo de envelhecimento humano, na perspectiva do reconhecimento das potencialidades do idoso, favorecendo a tomada de decisões.
Por outro lado, os idosos devem ser estimulados a terem autonomia e independência por meio de um processo contínuo de educação, crescimento, atualização e realização pessoal, de forma a incluí-los como membros ativos e construtores da sociedade. O envelhecimento saudável deve ter caráter abrangente, reconhecendo que os cuidados com a saúde e a integridade física são tão importantes quanto a participação ativa em relações sociais, econômicas, culturais, espirituais e civis. ${ }^{27}$

A contribuição do enfermeiro advém do desenvolvimento de ações conjuntas, com o idoso, o familiar e o cuidador, visando incentivar a expressão de desejos e capacidade de decisão nesta etapa da vida.

Concernente à análise espacial, os mapas temáticos, evidenciaram heterogeneidade da distribuição espacial dos menores escores nos domínios e facetas de qualidade de vida. A abordagem a partir da localização pontual dos eventos permitiu a identificação de áreas de maior concentração em que estão localizados os idosos com menores escores de qualidade de vida.

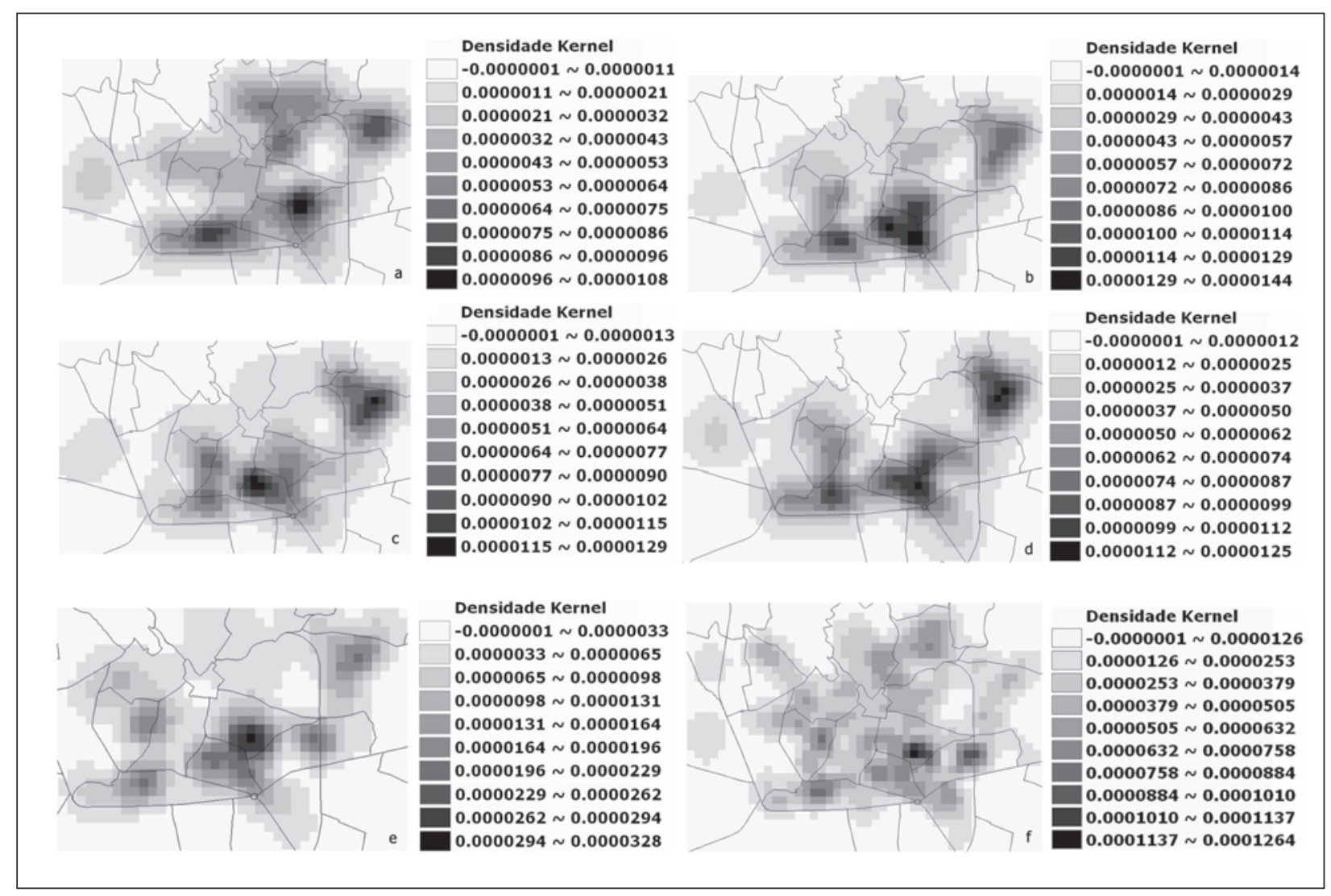

Figura 1 - Mapas da distribuição da densidade de idosos segundo menores escores nos domínios do WHOQOL-BREF, sobrepostos pelos bairros: (a) físico, (b) psicológico, (c) relações sociais, (d) meio ambiente, (e) qualidade de vida geral e (f) total de entrevistados. Uberaba-MG, 2010 
A figura 1a evidencia que os menores escores de qualidade de vida no domínio físico encontraram-se no bairro São Vicente, sul do Abadia, seguido pelo Parque das Américas e Jardim Elza Amuí (I, II e III), em menor densidade. Este domínio, dentre outros fatores, apresenta relação com a dependência para tratamentos de saúde. ${ }^{10}$ Nestas localidades existem Unidades de Saúde Pública, no entanto, seria necessário novas investigações com o intuito de identificar o acesso dos idosos à esses serviços, assim como a sua adesão ao tratamento.

O idoso com dificuldade de ter suas necessidades de saúde atendidas nos serviços públicos, poderá apresentar comprometimento na sua saúde e, por sua vez, na qualidade de vida. Neste estudo, os maiores percentuais foram para idosos com baixa renda, o que dificulta a sua adesão à medicina de grupo. Verificou um baixo percentual de sujeitos com planos de saúde na Vila São Vicente $(8,7 \%)$, no Jardim Elza Amuí $(18,2 \%)$, nas Vilas Santo Antônio e Industrial $(26,1 \%)$ e no Parque das Américas $(27,7 \%){ }^{28}$

Evidenciou-se aglomerado com menor escore de qualidade de vida no domínio psicológico na região sul do bairro Abadia, Cidade Jardim e Vila Nossa Senhora Abadia (Figura 1b). Em menor intensidade foi observado aglomerado na Vila Inês Helena e norte do Parque das Américas (Figura 1b). Este domínio refere-se à aprendizagem, concentração, autoestima e espiritualidade.${ }^{10} \mathrm{Os}$ idosos da presente pesquisa apresentam baixa escolaridade, que pode influenciar este domínio, sendo agravado pela ausência da cobertura da Estratégia Saúde da Família (ESF) nos bairros Cidade Jardim, Jardim Pinheiros e Vila São Marcos. ${ }^{29}$

Embora a relações sociais tenha obtido o maior escore de qualidade de vida entre os idosos, observam-se aglomerados de menores escores deste domínio nos bairros Cidade Jardim, Vila São Marcos e Jardim Elza Amuí (II e III) (Figura 1c). Nestas localidades a falta de suporte social repercute negativamente na qualidade de vida dos idosos. Destaca-se que no Jardim Elza Amuí, a ESF não acompanha todas as famílias $(40,4 \%)$, devido ao número reduzido de agentes comunitários de saúde, podendo associar-se à menor percepção de apoio ao idoso. As demais localidades não possuem cobertura da ESF. ${ }^{29}$

$\mathrm{Na}$ figura 1d, observa-se que os bairros Leblon, sudeste do Abadia, centro do Parque das Américas, Jardim Elza Amuí (I, II e III) e Cidade Nova apresentaram aglomerados de menores escores no domínio meio ambiente. Este domínio avalia a segurança física, proteção, ambiente no lar, recursos financeiros, cuidados de saúde e sociais, participação e oportunidade de lazer e ambiente físico. ${ }^{10}$

No bairro Leblon os menores escores podem estar relacionados ao mau estado de conservação das praças e problemas como a falta de abrigos nos pontos e áreas sem itinerário de transporte público. ${ }^{29}$ No bairro Abadia não há posto policial, a praça pública está desarborizada e, segundo a população, falta segurança pública, principalmente próximo às escolas, e verifica-se o uso de drogas ilícitas em vias públicas. ${ }^{29}$ Destaca-se que nas localidades do Jardim América existem chefes de família sem escolaridade $(9 \%)$ e renda mensal familiar inferior a $\mathrm{R} \$ 60,00(3,9 \%){ }^{29}$

Quanto às regiões do Jardim Elza Amuí (I, II, III, IV) e Cidade Nova são áreas em desenvolvimento, contando com construções inacabadas, gerando acúmulo de poeira nas residências. Encontram-se ainda lotes vagos (20\%), utilizados como depósito de entulhos, colaborando para a presença de animais peçonhentos e roedores. ${ }^{29}$

Os menores escores de qualidade de vida geral estão fortemente localizados na região sul do bairro Abadia, (Figura 1e). Referente à densidade dos idosos entrevistados no município, observa-se poucos aglomerados na região sul da Abadia e junção da Vila Esperança com Bairro de Lourdes (Figura 1f). 


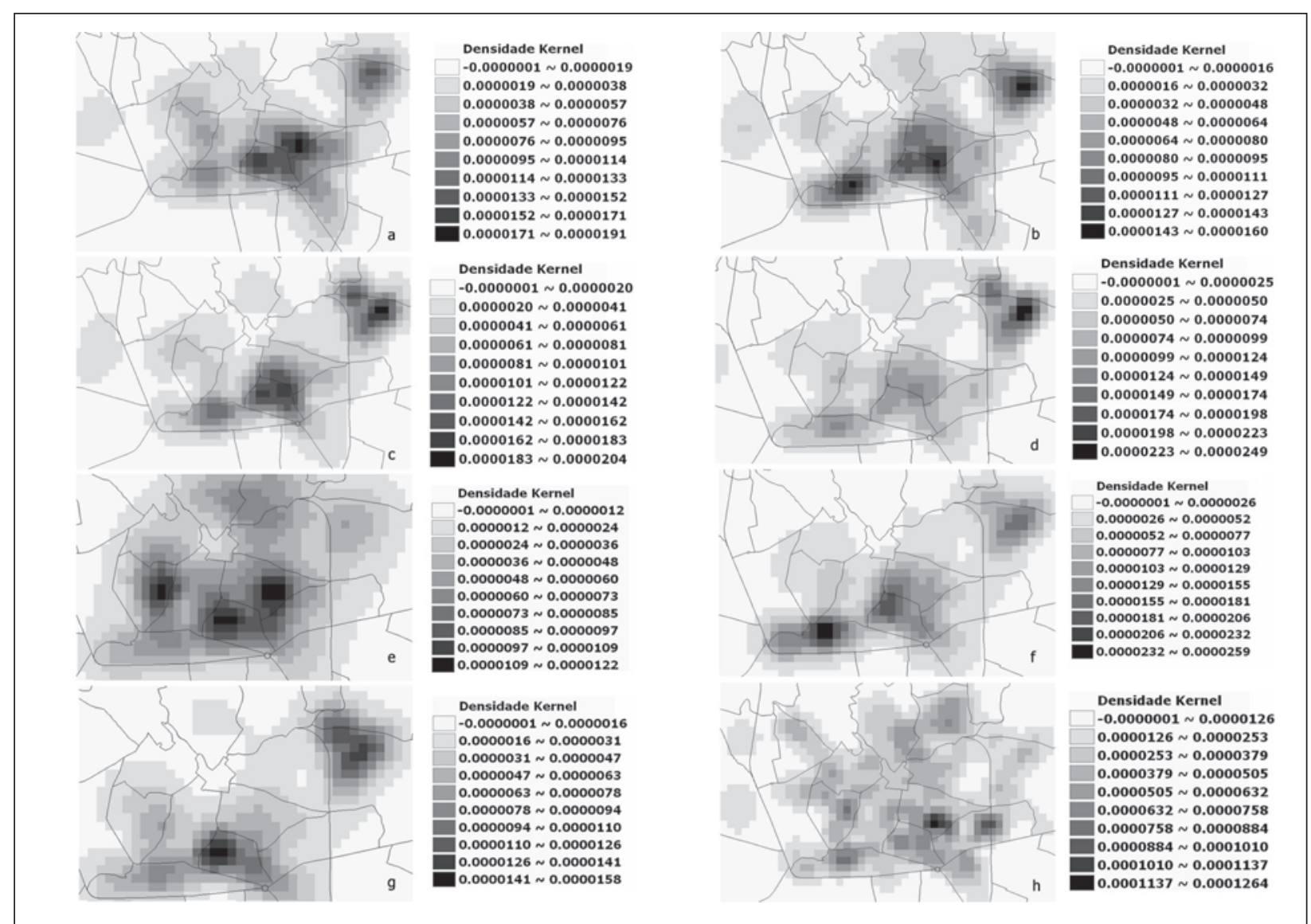

Figura 2 - Mapas da distribuição da densidade de idosos segundo menores escores nas facetas do WHOQOL-OLD, sobrepostos pelos bairros: (a) funcionamento dos sentidos, (b) autonomia, (c) atividades passadas, presentes e futuras, (d) participação social, (e) morte e morrer, (f) intimidade, (g) escore total e (h) total de entrevistados. Uberaba-MG, 2010

Os maiores aglomerados de menores escores de qualidade de vida da faceta funcionamento dos sentidos foram encontrados nos bairros Cidade Jardim, Vila São Marcos, Jardim Pinheiros, sul do Abadia e Vila São Vicente (Figura 2a).

Os menores escores de qualidade de vida na faceta autonomia encontraram fortes aglomerados nos bairros periféricos Vila Inês Helena, norte do Parque das Américas, Leblon, sul do Abadia, Jardim Elza Amuí (I, II, II e IV) e Residencial Mangueiras (Figura 2b).

As regiões do Jardim Elza Amuí (I, II, II e IV) e Residencial Mangueiras apresentaram aglomerados com menores escores de qualidade de vida na faceta atividades passadas, presentes e futuras, Figura 2c. Em menor densidade, os bairros Leblon e Cidade Jardim (Figura 2c).

Fortes aglomerados foram obtidos no Jardim Elza Amuí (I, II e II) e Residencial Mangueiras referente aos menores escores de qualidade de vida na participação social (Figura 2d). Este fato pode estar relacionado à ausência de espaços de lazer e opções culturais. Além disso, aproximadamente, $70 \%$ da comunidade não participa de nenhum tipo de atividade devido a escassez de opções. ${ }^{29}$

Os menores escores de qualidade devida na faceta morte e morrer apresentaram-se nos bairros Cidade Jardim, Vila São Marcos, Jardim Pinheiros, Vila Santa Maria, Leblon e leste do Abadia (Figura 2e). Esta faceta avalia as preocupações e temores relacionados à morte. ${ }^{11}$ Destaca-se que a não cobertura pela ESF pode ser um dificultador para que o idoso tenha um local no qual possa discutir estas questões e vivenciar esta etapa da vida de modo mais tranquilo. ${ }^{29}$

$\mathrm{O}$ aglomerado mais forte, referente aos menores escores de qualidade de vida na faceta intimidade, foi entre os idosos residentes nos bairros periféricos Vila Raquel, Vila Inês Helena, Vila Silva Campos e Parque das Américas (Figura 2f).

Os maiores aglomerados, no escore total, foram nos bairros: Cidade Jardim, Vila São Marcos, Parque 
Bom Retiro e Jardim Pinheiros (Figura 2g). Em menor densidade, Jardim Elza Amú (I e III) (Figura 2g). A densidade dos idosos entrevistados no município evidencia poucos aglomerados (Figura $2 \mathrm{~h}$ ).

A aplicação do SIG nesta pesquisa contribuiu para identificar as especificidades de atenção aos idosos, nas diversas localidades do município, favorecendo a reorganização e qualificação dos serviços de saúde.

\section{CONCLUSÕES}

A maioria era do sexo feminino, com faixa etária entre 60 e 70 anos, casados ou que moravam com companheiro, de quatro a oito anos de estudo e renda individual de um salário mínimo. Concernente à qualidade de vida, os maiores escores foram para o domínio relações sociais e a faceta funcionamento dos sentidos, enquanto os menores estavam relacionados ao físico e a autonomia.

A distribuição espacial dos menores escores de qualidade de vida apresentou aglomerados nas regiões periféricas do município. Porém, ressalta-se como limitação deste estudo o fato de não permitir identificar os fatores associados aos menores escores nestas localidades. Diante disso, faz-se necessário novas investigações visando aprofundar sobre os fatores que podem estar contribuindo para estes resultados, sejam eles de saúde; ambientais; econômicos; sociais ou culturais. Nos bairros com menores escores de qualidade de vida é necessária uma ação conjunta dos serviços de saúde, da comunidade e de outros equipamentos sociais presentes na localidade com vistas à racionalização das ações e proposições de estratégias de intervenção.

\section{REFERÊNCIAS}

1. Veras R. Envelhecimento populacional contemporâneo: demandas, desafios e inovações. Rev Saúde Pública. 2009 Abr; 43(3):548-54.

2. Instituto Brasileiro de Geografia e Estatística (IBGE). Síntese de indicadores sociais: uma análise das condições de vida da população brasileira 2009. Brasília (DF): Ministério do Planejamento, Orçamento e Gestão. [acesso 2010 Mar 31]. Disponível em: http:/ /www.ibge.gov.br./home/

3. Datasus [página da internet]. Brasília (DF): MS; 2009 [acesso 2009 Abr 27]. Disponível em: http:/ / tabnet.datasus.gov.br/tabdata/cadernos/ cadernosmap.htm.

4. Fleck MPA, Chachamovich E, Trentini C. Projeto WHOQOL-OLD: método e resultados de grupos focais no Brasil. Rev Saúde Pública. 2003 Dec; 37(6):793-9.

5. WHOQOL Group. The world health organization quality of life assessment (WHOQOL): position paper from the world health organization. Soc Sci Med. 1995 Nov; 41(10):1403-9.

6. Barcellos CC, Sabroza PC, Peiter P, Rojas LI. Organização espacial, saúde e qualidade de vida: análise espacial e uso de indicadores na avaliação de situações de saúde. Informe Epidemiológico do SUS. 2002 Jul-Set; 11(3):129-38.

7. Moss MP, Schell MC, Goins RT. Using GIS in a first national mapping of functional disability among older American Indians and Alaska natives from the 2000 census. Int J Health Geogr. 2006 Set; 5(37).

8. Magalhães RM, Corrente JE, Ruiz T, Simões LB. Distribuição espacial dos idosos segundo seus conceitos de qualidade de vida. Rev APS. 2008 OutDez; 11(4):374-9.

9. Tavares DMS, Iwamoto HH, Miranzi SSC, Rodrigues LR, Machado ARM, Pereira GA. Condições de saúde de idosos residentes no município de Uberaba [Relatório final]. Uberaba (MG): Universidade Federal do Triângulo Mineiro; 2007

10. Chachamovich E, Fleck MPA. Desenvolvimento do WHOQOL BREF. In: Fleck MPA, organizador. A avaliação de qualidade de vida: guia para profissionais da saúde. Porto Alegre (RS): Artmed; 2008. p.74-82.

11. Chachamovich E, Trentini CM, Fleck MPA, Schmidt $S$, Power M. Desenvolvimento do instrumento WHOQOL-OLD. In: Fleck MPA, organizador. A avaliação de qualidade de vida: guia para profissionais da saúde. Porto Alegre (RS): Artmed; 2008. p.102-11.

12. Ministério da Saúde (BR). Secretaria de Vigilância em Saúde. Fundação Oswaldo Cruz. Introdução à estatística espacial para a saúde pública. Brasília (DF): Ministério da Saúde; 2007.

13. Portal Brasil. Salário mínimo. [acesso 2011 Abr 06]. Disponível em: http://www.portalbrasil.net/ salariominimo.htm

14. Portal Brasil. US\$Dólar, Euro e risco país. [acesso 2011 Abr 06]. Disponível em: http://www. portalbrasil.net/2008/economia/dolar_riscopais_ dezembro.htm

15. Tavares DMS, Pereira GA, Iwamoto HH, Miranzi SSC, Rodrigues LR, Machado ARM. Incapacidade funcional entre idosos residentes em um município do interior de Minas Gerais. Texto Contexto Enferm. 2007 Jan-Mar; 16(1):32-9.

16. Joia LC, Ruiz T, Donalisio MR. Condições associadas ao grau de satisfação com a vida entre a população de idosos. Rev Saúde Pública. 2007 Fev; 41(1):131-8.

17. Carvalho JAM, Rodríguez-Wong LL. A transição da estrutura etária da população brasileira na primeira 
metade do século XXI. Cad Saúde Pública. 2008 Mar; 24(3):597-605.

18. Caetano JA, Costa AC, Santos ZMSA, Soares E. Descrição dos fatores de risco para alterações cardiovasculares em um grupo de idosos. Texto Contexto Enferm [online]. 2008 Jun [acesso 2010 Mar 31]; 17(2):327-35. Disponível em: http:// www.scielo.br/scielo.php?script=sci_arttext\&pid =S0104-07072008000200015.

19. Trentini CM, Chachamovich E, Figueiredo $M$, Hirakata VN, Fleck MPA. A percepção de qualidade de vida do idoso avaliada por si próprio e pelo cuidador. Estud Psicol. 2006 Mai-Ago; 11(2):191-7.

20. Pereira RJ, Cotta RMM, Franceschini SCC, Ribeiro RCL, Sampaio RF, Priore SE, et al. Contribuição dos domínios físico, social, psicológico e ambiental para a qualidade de vida global de idosos. Rev Psiquiatr Rio Gd Sul. 2006 Abr; 28(1):27-38.

21. Campos FG, Barrozo LV, RUIZ T, César CLG, Barros MBAZ, Carandina L, et al. Distribuição espacial dos idosos de um município de médio porte do interior paulista segundo algumas características sóciodemográficas e de morbidade. Cad Saúde Pública. 2009 Jan; 25(1):77-86.

22. Silva MJ, Lopes MVO, Araújo MFM, Moraes GLA. Avaliação do grau de dependência nas atividades de vida diária em idosos da cidade de Fortaleza-Ceará. Acta Paul Enferm. 2006 Abr-Jun; 19(2):201-6.

23. Moraes NAS, Witter GP. Velhice: qualidade de vida intrínseca e extrínseca. Boletim de Psicologia, 2007
Jul-Dez; 57(127):215-38.

24. Tokuda Y, Jimba M, Yanai H, Fujii S, Inoguchi $\mathrm{T}$. Interpersonal trust and quality-of-life: a crosssectional study in Japan. PLoS ONE [online] 2008 Dez [acesso 2009 Jul 03]; 3(12):3985. Disponível em: http://www.plosone.org/article/ info:doi\%2F10.1371\%2Fjournal.pone.0003985.

25. Celich KLS. Domínios de qualidade de vida e capacidade para a tomada de decisão em idosos participantes de grupos de terceira idade [tese]. Porto Alegre (RS): Pontifícia Universidade Católica Rio Grande do Sul; 2008.

26. Ramos LJ. Variação do estado nutricional, da qualidade de vida e da capacidade de tomar decisão de idosos institucionalizados e nãoinstitucionalizados no município de Porto Alegre, RS [trabalho de conclusão de curso]. Porto Alegre (RS): Pontifícia Universidade Católica Rio Grande do Sul; 2008.

27. Inouye K, Pedrazzani ES. Nível de instrução, status socioeconômico e avaliação de algumas dimensões da qualidade de vida de octogenários. Rev Latinoam Enfermagem. 2007 Set-Out; 15(Esp):742-7.

28. Sistema de Informação da Atenção Básica - SIAB. Secretaria Municipal de Saúde. Consolidado das famílias cadastradas. Uberaba (MG): SMS; 2008.

29. Secretaria Municipal de Saúde. Departamento de Atenção Básica. Diagnóstico local do Plano Diretor da Atenção Primária à Saúde - Uberaba/MG. Uberaba (MG): SMS. 2010. 